\title{
Comparative Study of Absorbed Doses in Different Phantom Materials and Fabrication of a Suitable Phantom
}

\author{
M. A. Rahman', Md. Tofajjol Hoseen Bhuiyan ${ }^{2 *}$, M. M. Rahman ${ }^{3}$, M.N. Chowdhury ${ }^{4}$ \\ ${ }^{1}$ Scientific Officer, Designated Reference Institute for Chemical Measurement (DRiCM), BCSIR, Dhaka-1205, BANGLADESH \\ ${ }^{2}$ Lecturer, Department of Physics, Pabna University of Science and Technology, Rajapur, Pabna-6600, BANGLADESH \\ ${ }^{3}$ Research Officer, Khwaja Yunus Ali Medical College \& Hospital, Cancer Centre, Enayetpur, Sirajganj, BANGLADESH \\ ${ }^{4}$ Lecturer, Department of Basic Sciences and Humanities, University of Asia Pacific, Dhanmondhi, Dhaka, BANGLADESH \\ *Email for Correspondence: thbapon@gmail.com
}

\begin{abstract}
In Cancer treatment (radiotherapy) centers, Phantom is important in Quality Assurance routine check and absolute dosimetry conformation. Water is IAEA standard phantom material. But it has some technical difficulty in practical uses and other solid phantoms such as Polystyrene, PMMA is very expensive and locally not available. Hence, the purpose of this paper is to find out a phantom that will be technically very sound, cost effective and locally available. This study reveals that paraffin wax, which has some approximately similar properties (i.e. chemical composition, mass density and number of electrons/gram) to water, can be used as alternative of solid water phantom because of their proximity to the dose absorption property of water which is even better than some of the conventional solid phantoms used in radiation dosimetry. It is also found that paraffin wax phantom with air-bubble inside behaves differently to the radiation absorbing dose and therefore in dose absorption and dose conversion (scaling) factor.
\end{abstract}

Key words: Phantom, Dosimetry, Absorbed dose, Paraffin-wax, Scaling factor

This article is is licensed under a Creative Commons Attribution-NonCommercial 4.0 International License.

Attribution-NonCommercial (CC BY-NC) license lets others remix, tweak, and build upon work non-commercially, and although the new works must also

acknowledge \& be non-commercial.

\section{INTRODUCTION}

In radiotherapy treatment, dose delivery to the patient involves many steps, parameters, and factors. As a result, more complex quality controls (routine check and dosimetry) are required during the radiotherapy process to ensure that each step has as less error as possible. For this reason, before any radiation exposure to a patient, the radiation dose that to be delivered has to be planed and checked carefully. Dose distribution inside a patient is a very complex task as this cannot be measured in the patient's body directly. Hence, a patient's body needs to be replaced by a tissue-equivalent material. This material is commonly called 'phantom'. Its absorption and scattering properties should be as close to the human body as possible and also economically viable. Since the main component of body tissue is water, it has been widely used in many investigations. To carry out absolute dosimetry, a water phantom is needed. Water is recommended as the reference medium for the measurements of absorbed dose; both for photon and electron beam. The International Commission on Radiation Units and Measurements (ICRU) suggests that the uncertainty on dose measurement must be $\pm 5 \%$ (ICRU, 1976).

Water phantoms, however, are inconvenient to use in the Linac or ${ }^{60} \mathrm{Co}$ photon beams because it is difficult and timeconsuming to positioning or align it with the radiation beam. There is also difficulty in maintaining temperature and humidity of the water phantom and its surroundings. Because of these problems, in modern cancer clinics, water phantoms are replaced by water-equivalent (i.e. tissue-equivalent) solid phantoms (M Allahverdi, 1999). The conventional solid phantoms are solid water, polystyrene, acrylics and more. But these solid phantoms are very expensive and locally not available. Modern cancer clinics, especially in developing countries, have long been trying to get a tissue equivalent material (phantom), which will be locally available, inexpensive and equally excellent in quality. 
In our study, we were looking for cheap substances having properties similar to those of water. Paraffin wax is found to have density and electron density similar to those of water (E. William, 1999). Because of this water like properties; we decided to fabricate a phantom using paraffin wax. To test the suitability of the phantom we planned to determine the dose in the iso-center of the cube and compare it with that in water phantom and, therefore, calculate the scaling factor. A number (i.e., scaling factor) close to 1 is evident to be a good phantom.

We also planned to determine such scaling factors for widely used solid phantoms using water as a reference. We decided to make a comparison of these values and assess the suitability of the materials. These scaling factors may be used as a correction factor (F. M. Khan, 1994) whenever any material other than water is used as a phantom.

\section{MATERIALS AND METHOdS}

The present study is carried out using the facilities of the cancer center of the Khwaja Yunus Ali Medical College and Hospital (KYAMCH), Enayetpur, Sirajganj, Bangladesh. An Elektra Synergy S Linear Accelerator capable of delivering photon beam of three different energies $(4 \mathrm{MV}, 6 \mathrm{MV}, 15 \mathrm{MV})$ and electron beam of 5 different energies $(6 \mathrm{MeV}, 8 \mathrm{MeV}$, $10 \mathrm{MeV}, 12 \mathrm{MeV}, 15 \mathrm{MeV}, 18 \mathrm{MeV}$ ) (Elekta Synergy, 2014) has been used as the source of mono-energetic photon beams of energy $6 \mathrm{MV}$ and $15 \mathrm{MV}$. Paraffin wax, solid water and PMMA are examined for their suitability as alternative phantoms for routine quality assurance of the irradiation system. Their dose absorptions along the central axis of the photon beam have been compared with that in water, an IAEA standard phantom material. Absorbed dose based absolute dosimetry protocols TG-51and IAEA TRS-398 have been used for all these measurements. Scaling factors for dosimetry of the materials are determined. These factors can be used as correction factors.

Paraffin wax is mostly found as a white, odorless, tasteless, waxy solid, with a typical melting point between about $46^{\circ} \mathrm{C}$ and $68{ }^{\circ} \mathrm{C}\left(115^{\circ} \mathrm{F}\right.$ and $\left.154^{\circ} \mathrm{F}\right),\left(\mathrm{E}\right.$. William, 1999) and having a density of around $0.9 \mathrm{~g} / \mathrm{cm}^{3}$. It is insoluble in water, but soluble in ether, benzene, and certain esters. Paraffin is unaffected by most common chemical reagents but burns readily.

In our study different phantom materials were used. Their names along with their properties are presented in Table1.

Table 1: Phantom material properties

\begin{tabular}{|c|c|c|c|}
\hline Material name & Chemical Composition & Mass density $\left(\mathrm{gm} / \mathrm{cm}^{3}\right)$ & Number of Electrons $/ \mathrm{g}\left(\times 10^{23}\right)$ \\
\hline Paraffin wax & $\mathrm{C}_{\mathrm{n}} \mathrm{H}_{2 \mathrm{n}+2,}, 20 \leq \mathrm{n} \leq 40$ & $0.88-0.92$ & 3.44 \\
\hline Solid water & Epoxy resin-based mixture & 1.00 & 3.34 \\
\hline PMMA & $\left(\mathrm{C}_{5} \mathrm{O}_{2} \mathrm{H}_{8}\right)_{\mathrm{n}}$ & $1.16-1.20$ & 3.24 \\
\hline Water & $\mathrm{H}_{2} \mathrm{O}$ & 1.00 & 3.34 \\
\hline
\end{tabular}

We used two different techniques to make cubic phantom with paraffin wax: at the very first, we bought some paraffin wax from local market and made a cubic phantom of $20 \mathrm{~cm}$ a side by pouring melted paraffin wax into a steel made cubic dice (J. C. Huang, fall 2000). Secondly, we bought some readily available paraffin wax slabs of dimension $30 \times 25 \times 4 \mathrm{~cm}^{3}$ from the local market and cut them into a size of $21 \times 21 \times 4 \mathrm{~cm}^{3}$ and then joined them together by pressing them after slightly heated on a hot plate at $40^{\circ} \mathrm{C}$ and finally had a fine cubic phantom of $20 \mathrm{cma}$ side. To insert an ionization chamber at the iso-center of the phantom, each phantom was drilled along a vertical line with much care.

To look the inside image of these fabricated wax phantoms, they were placed in the Philips CT-Scanner [Model no: Philips Brilliance CT 64-channel scanner] of the cancer center of the KYAMCH. It was found that the first Phantom had lots of air bubbles whereas second one had no air bubble inside shown in figure-1.

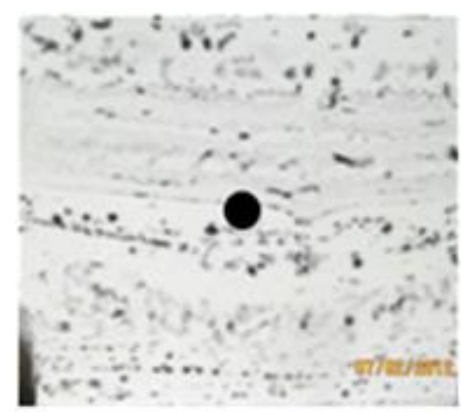

Phantom-1

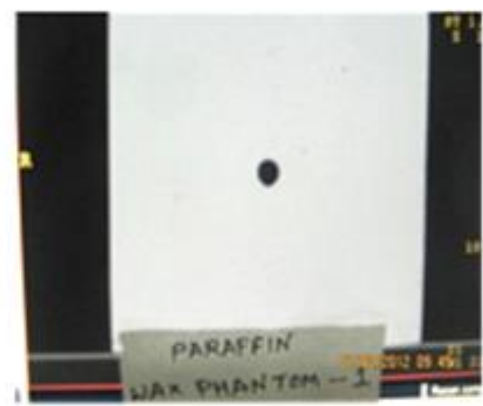

Phantom-2

Fig. 1: CT-scan images of the paraffin wax phantom

The paraffin wax phantom was then placed on the couch of the linear accelerator (Elektra Synergy S Linac).The phantom surface center was aligned with the central axis of the beam from the gantry at zero degree angles. The 
distance between the phantoms surfaces to the source (i.e. SSD) was made $100 \mathrm{~cm}$ with the help of optical mark reader by moving the couch vertically. After positioning the phantom, a Farmer $(0.6 \mathrm{cc})$ type ionization chamber [PTW Farmer chamber type 30013] was inserted to the hole. The other end of the ionization chamber was connected to an electrometer [PTW UNIDOS]. The monitor unit of the linear accelerator was set 100 and after closing the door of the accelerator room radiation was initiated. Then the electrometer readings were taken. Readings were recorded for all the four faces of the cube, facing radiation source (linear accelerator). The readings were repeated three times for each of $6 \mathrm{MV}$ and $15 \mathrm{MV}$ photon beams. The solid water phantom and water phantom were also placed on the couch of the Linac and readings were taken for $6 \mathrm{MV}$ and $15 \mathrm{MV}$ in a similar fashion.

For only wax phantoms, readings were taken for four faces up. For the other phantoms only one surface was irradiated. The temperature and pressure of all phantoms were taken by the digital thermometer and barometer.

Absorbed dose $D_{W, Q}$ to water at the reference depth, $\mathrm{Zref}$, in a water phantom irradiated by a beam of quality $Q$ is

$D_{W, Q}=M_{Q} \times N_{D, W} \times K_{T P} \times K_{S} \times K_{p o l} \times K_{Q, Q_{o}}$

where, $M_{Q}=$ Monitor reading.

$N_{D, W}=$ Calibration factor regarding absorbed dose to water.

$K_{T P}=$ Factor to correct the response of an ionization chamber for the effect of the difference that may exist between the standard reference temperature and pressure specified by the standards laboratory and the temperature and pressure of the chamber in the user facility under different environmental conditions.

$K_{S}=\quad$ Factor to correct the response of an ionization chamber for the lack of complete charge collection (due to ion recombination).

$K_{p o l}=$ Factor to correct the response of an ionization chamber for the effect of a change in polarity of the polarizing voltage applied to the chamber.

$K_{Q, Q_{0}}=$ Factor to correct for the difference between the response of an ionization chamber in the reference beam

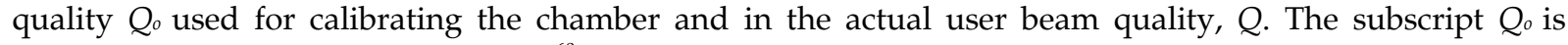
omitted when the reference quality is ${ }^{60} \mathrm{Co}$ gamma radiation (i.e. the reduced notation $k_{Q}$ always corresponds to the reference quality ${ }^{60} \mathrm{Co}$ ).

To determine polarity correction factor, readings of the electrometer were taken with positive and negative polarity. The collected charges $M_{1}$ and $M_{2}$ at the polarizing voltages $V_{1}$ and $V_{2}$ were measured, where $V_{1}$ was the normal operating voltage and $V_{2}$ a lower voltage; the ratio $V_{1} / V_{2}$ should ideally be equal to or larger than three. In the above experiment absorbed dose based absolute dosimetry protocols TG-51and IAEA TRS 398 were followed.

\section{RESULTS AND Discussion}

The dosimetry property of paraffin wax was studied and compares with those of solid water phantoms and PMMA phantoms used widely in cancer clinics in place of water phantoms. Scaling factors (Jan Seuntjens, 2005) and absorbed dose for different types of phantoms were calculated for both $6 \mathrm{MV}$ and 15MV photon beams and were summarized in Table 2 and Table 3. Water phantom is referred as a standard for absolute dosimetry and scaling factor is unity for water phantom.

Table 2: Scaling factor of different phantom materials for $6 \mathrm{MV}$ photon beam

\begin{tabular}{|l|c|c|c|c|c|}
\hline \multirow{2}{*}{ Function } & \multicolumn{4}{|c|}{ Name of Phantom } \\
\cline { 2 - 6 } & $\begin{array}{c}\text { Water } \\
\text { phantom }\end{array}$ & $\begin{array}{c}\text { Paraffin wax-1 having } \\
\text { air bubble }\end{array}$ & $\begin{array}{c}\text { Paraffin } \\
\text { wax-2 }\end{array}$ & $\begin{array}{c}\text { Solid } \\
\text { water }\end{array}$ & PMMA \\
\hline Absorbed dose & 0.6892 & 0.7051 & 0.6948 & 0.6758 & 0.6691 \\
\hline Scaling factor & 1 & 0.977 & 0.992 & 1.020 & 1.030 \\
\hline Deviation from water phantom & 0 & $-2.3 \%$ & $-0.8 \%$ & $+2.0 \%$ & $+3.0 \%$ \\
\hline
\end{tabular}

Table 3: Scaling factor for different phantom materials for $15 \mathrm{MV}$ photon beam

\begin{tabular}{|l|c|c|c|c|c|}
\hline \multirow{2}{*}{ Function } & \multicolumn{4}{|c|}{ Name of Phantom } \\
\cline { 2 - 6 } & $\begin{array}{c}\text { Water } \\
\text { phantom }\end{array}$ & $\begin{array}{c}\text { Paraffin wax-1 having } \\
\text { air bubble }\end{array}$ & $\begin{array}{c}\text { Paraffin } \\
\text { wax-2 }\end{array}$ & $\begin{array}{c}\text { Solid } \\
\text { water }\end{array}$ & PMMA \\
\hline Absorbed dose & 0.8142 & 0.8350 & 0.8042 & 0.7688 & 0.7897 \\
\hline Scaling factor & 1 & 0.975 & 1.012 & 1.059 & 1.031 \\
\hline Deviation from water phantom & 0 & $-2.5 \%$ & $+1.2 \%$ & $+5.9 \%$ & $+3.1 \%$ \\
\hline
\end{tabular}


All other phantoms show deviations in the scaling factors from the value of water phantom (i.e., from 1) and are graphically represented by figure 2 and figure 3 . For both energies, it is obvious from the figures that the deviation is least for the paraffin wax phantom compare to the other phantom. And paraffin wax-2 phantom is more close to the water phantom value than the paraffin wax-1 phantom (i.e. the deviation is slightly higher for the wax phantom having air bubbles inside).

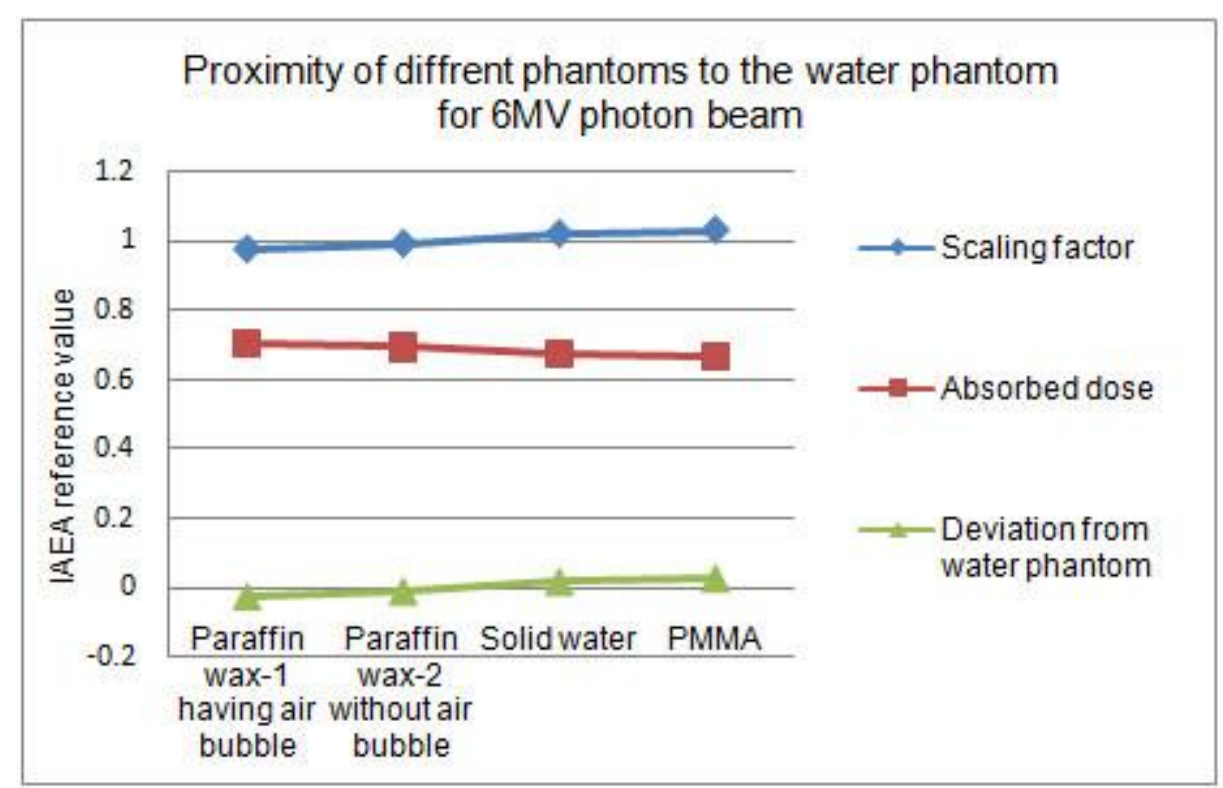

Fig. 2: Graphical view of scaling factor, absorbs dose and the deviation of scaling factor from IAEA standard value for different phantom materials at $6 \mathrm{MV}$ radiation energy

Also, the deviation is above 5\% for solid water phantom for $15 \mathrm{MV}$ photon beam and for PMMA phantom, the deviation is $3.0 \%$ for $6 \mathrm{MV}$ and $3.1 \%$ for $15 \mathrm{MV}$. Since phantom material with less deviation indicates more suitability for practical dosimetry, paraffin wax phantom would be an excellent alternative of water phantom. The picture is almost similar in case of absorbed dose: dose absorption of paraffin wax phantom is found very close to the water phantom unlike another phantom for both 6MV and 15MV energy beams. Deviations of absorbed dose from that in water phantom are not negligible for some solid phantoms, especially for PMMA and solid water phantom. In the daily quality assurance (AIP, 1994) (QA) of the machine output and absolute dosimetry, the scaling factor should be used as a correction factor for the solid phantoms.

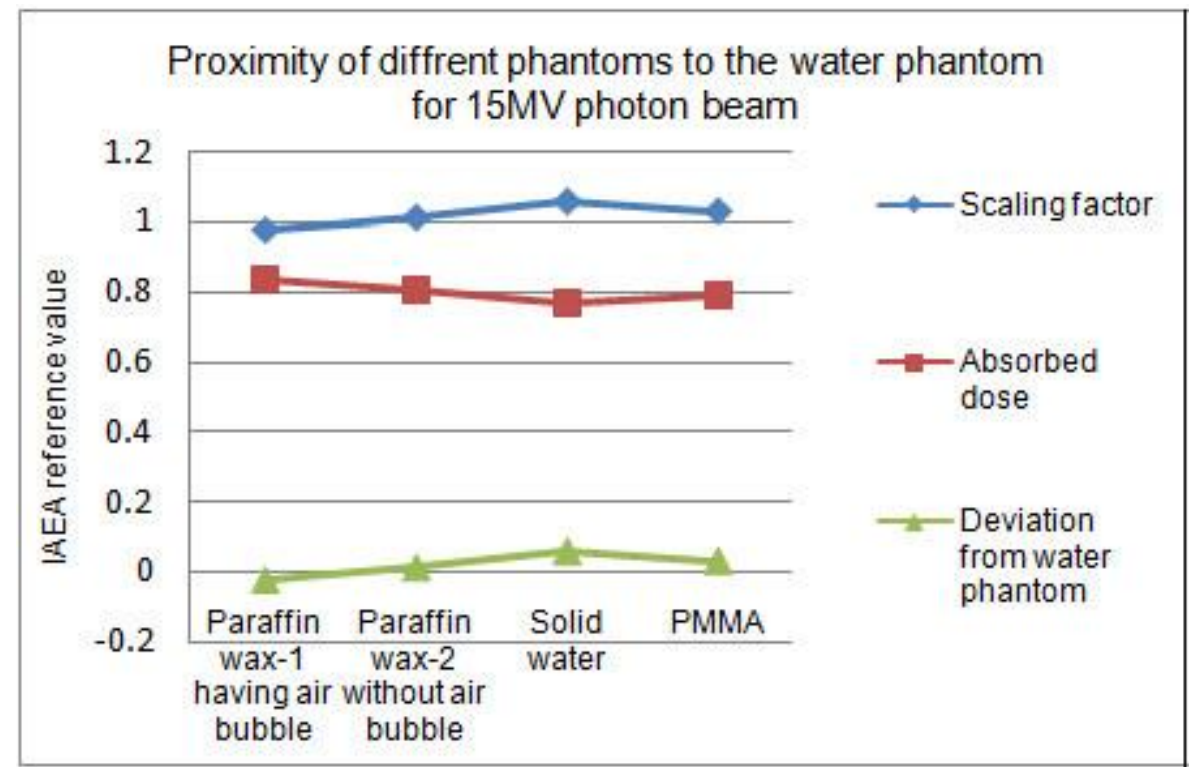

Fig. 3: Graphical view of scaling factor, absorbs dose and the deviation of scaling factor from IAEA standard value for different phantom materials at 15MV radiation energy 
From Figure 4, it can also be surmised that deviation in scaling factor is a function of radiation beam's energy and phantom materials: deviation in scaling factor increases with the increment of radiation beam's energy and the value of increment of deviation in scaling factor is different for different phantom material.

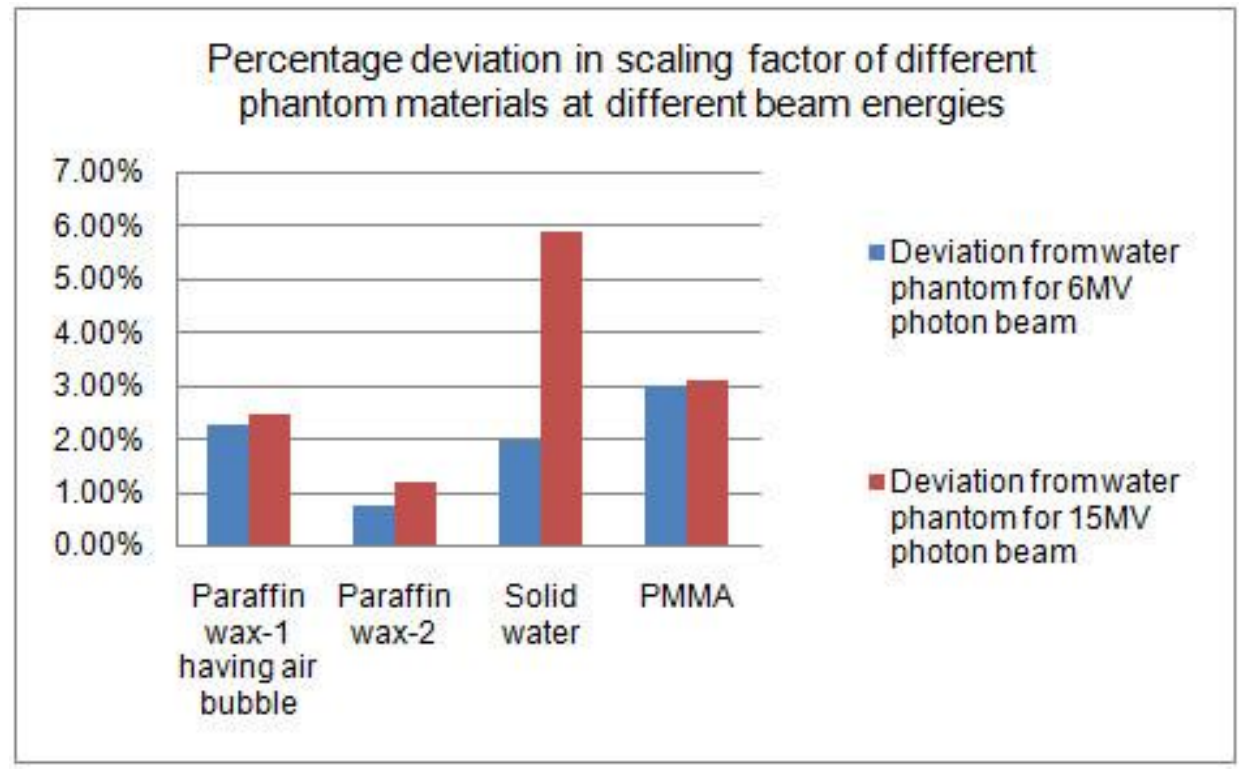

Fig. 4: Comparison of scaling factors for different phantom materials at different beam energies

\section{CONCLUSION}

Paraffin wax phantom can be used in radiotherapy centers for routine QA check and dosimetry confirmation in lieu of water phantom or other solid phantoms with comparatively higher accuracy and lowest time consumption. Paraffin wax phantom is much cheaper than the conventional solid phantoms, because it costs only $\$ 35$, whereas a solid water or PMMA phantom costs about $\$ 5000$. Moreover, it is readily available in local market. However, the durability of the paraffin wax phantom was not tested in our study and need to be studied further. It might be possible to extend the life time of paraffin wax phantom by using plastic frames at the edges of the wax cube. We consider our simple research work presented here as a valuable addition to radiotherapy treatment phenomenology that can arouse interest in practical researchers.

\section{REFERENCES}

Almond PR, Biggs PJ, Coursey BM, et al. (1999) AAPM's TG-51 protocol for clinical reference dosimetry of high energy photon and electron beams. Med Phys 26(9):1847-1870

America Association of Physicists in Medicine (1983) Task Group 21: A protocol for the determination of absorbed dose from high energy photon and electron beams. Med Phys 10,741

American Institute of Physics (1994) Physical Aspects of Quality Assurance in Radiation Therapy AAPM Report No. 13 New York

D. Mihailescu, C. Borcia (2006) Water Equivalency of Some Plastic Materials Used in Electron Dosimetry:A Monte Carlo Investigation. Romanian Reports in Physics, Vol. 58, No. 4, P. 415-425

Elekta Synergy® S (2014) Image-guided stereotactic radiation therapy system http:/ / www.elekta.com/healthcareprofessionals/products/elekta-oncology/oncology-treatment-solutions/elekta-synergy/synergy-s.html

F. M. Khan (1994) The physics of radiation therapy. Second Edition. Williams \&Wilins, USA

IAEA, Technical Reports Series No. 277 (2nd edn in 1997) (1987). IAEA, Vienna

ICRU (1976) Determination of absorbed dose in a patient irradiated by beams of $x$ or gamma rays in radiotherapy procedures. International Commission on Radiation Units and Measurements ICRU, Report 24, Bethesda

International Atomic Energy Agency (1997) The use of plane parallel ionization chambers in high energy electron and photon beams: An international code of practice for dosimetry. Technical Reports series No. 381, IAEA, Vienna

International Commission On Radiation Units And Measurements (1989) Tissue Substitutes in Radiation Dosimetry and Measurement. Rep. 44, ICRU, Bethesda, MD

J. C. Huang, L. E. Reinstein (Fall 2000) Evaluation of an innovative plastic cube phantom designed to improve the efficiency of accelerator QA. Journal of Applied Clinical Medical Physics, Volume 1, Number 4

Jan Seuntjens,Marina Olivares, Michael Evans, Ervin Podgorsak (September 2005) Absorbed dose to water reference dosimetry using solid phantomsin the context of absorbed-dose protocols. Med Phys 32 (9), 0094-2405 
K. A. Gifford, D. S. Followill, H. H. Liu, G. Starkschall (2002) Verification of the Accuracy of a Photon DoseCalculation Algorithm. Journal of Applied Clinical Medical Physics, Vol. 3, No. 1, pp. 26-45

Klein EE, Hanley J, Bayouth J, et al. (2009) Task Group 142 report: quality assurance of medical accelerators. Med Phys 36:41974212

M Allahverdi, A Nisbet, D I Thwaites (1999) An evaluation of epoxy resin phantom materials for megavoltage photon dosimetry. Phys Med Biol. 44/1125

Nasser, E. William (1999) Waxes, Natural and Synthetic. New York: Marcel Dekker

Practical Course in Reference Dosimetry (January 2014) National Physical Laboratory MV Photon Dosimetry at NPL, UK

Technical Report Series no.398 (2000) Absorbed Dose Determination in External Beam Radiotherapy- An International Code of Practice for Dosimetry Based on Standards of Absorbed Dose to Water. International Atomic Energy Agency (IAEA), Vienna

--0 -- 